\title{
LA HUELLA DE LAS OSCILACIONES GLOBALES DEL NIVEL DEL MAR DURANTE LOS ÚLTIMOS 530000 AÑOS EN EL GOLFO DE LEÓN Y SU VARIABILIDAD DURANTE EL ESTADIO ISOTÓPICO MARINO 3
}

\author{
J. FRIGOLA ${ }^{1 *}$, M. CANALS ${ }^{1}$, I. CACHO ${ }^{1}$, A. MORENO ${ }^{2}$, \\ F.J. SIERRO ${ }^{3}$, J.A. FLORES ${ }^{3}$, J.O. GRIMALT ${ }^{4}$ \\ ${ }^{1}$ GRC en Geociències Marines, Dept. d'Estratigrafia, Paleontologia i Geociències Marines, \\ Universitat de Barcelona, Barcelona. \\ ${ }^{2}$ Instituto Pirenaico de Ecología, CSIC, Campus Aula Dei, Av. Montañana 1005, 50059 Zaragoza. \\ ${ }^{3}$ Departamento de Geología, Universidad de Salamanca. \\ ${ }^{4}$ Instituto de Diagnóstico Ambiental y Estudios del Agua, CSIC, Barcelona.
}

RESUMEN. El sondeo PRGL1-4, recuperado en el talud superior, ha proporcionado un registro único, continuo y de larga duración para la investigación del impacto de las oscilaciones del nivel del mar de escala glacial/interglacial durante el Pleistoceno Superior en la construcción del margen continental del Golfo de León, dominado por aportaciones sedimentarias de origen fluvial. El estudio en detalle de los cambios del tamaño de grano y la composición elemental del sedimento, junto con una robusta cronoestratigrafía basada en el registro de isótopos de oxígeno en foraminíferos planctónicos, ha permitido reinterpretar la sismoestratigrafia del margen para los últimos $530 \mathrm{ka}$. El sondeo PRGL1-4 comprende el apilamiento formado por cinco unidades depositadas durante las bajadas del nivel del mar de los últimos cinco ciclos glacial/interglacial de $100 \mathrm{ka}$. La extensa plataforma del Golfo de León ha jugado un papel determinante en el desarrollo del margen profundo puesto que las oscilaciones del nivel del mar, además de modificar el espacio de acomodación, influyeron directamente en cambios cíclicos del modelo deposicional a través de la activación y desactivación de procesos con gran capacidad erosiva y de transporte de sedimento más allá de la plataforma. El registro del sondeo PRGL1-4 también muestra el impacto de las oscilaciones del nivel del mar a escala milenaria durante el MIS 3, con evidencias claras de la ocurrencia de niveles del mar altos durante la fase inicial de todos y cada uno de los interestadios cálidos de los ciclos de Dansgaard-Oeschger. El registro PRGL1-4 supone, por tanto, la primera evidencia de un acoplamiento consistente entre las oscilaciones del nivel del mar y la variabilidad climática rápida de escala milenaria asociada a los ciclos de Dansgaard-Oeschger. 
The imprint of global sea level oscillations during the last 530000 years in the Gulf of Lion and its variability during Marine Isotope Stage 3

\begin{abstract}
Borehole PRGL1-4, drilled in the upper slope, provided an exceptional, continuous and long record for investigating the impact of glacial/interglacial sea level oscillations during the Upper Pleistocene in the outbuilding of the Gulf of Lion continental margin, which was dominated by fluvial sedimentary inputs. The detailed analysis of temporal oscillations in grain-size and geochemical elemental composition, together with a robust chronostratigraphy based on the oxygen isotopic record of planktonic foraminifera, allowed reinterpreting the seismostratigraphy of the margin for the last $530 \mathrm{ky}$. Borehole PRGL1-4 consists of five stacked units that formed during the sea level lowerings of the last five glacial/interglacial $100 \mathrm{ky}$ cycles. The areal extent of the Gulf of Lion's continental shelf has played a decisive role in the development of the margin since sea level oscillations, in addition of modifying accommodation space, cyclically modified the margin depositional pattern through the highstand activation and lowstand deactivation of highly erosion and transport processes able to carry large amounts of sediment beyond the shelf edge. The PRGL1-4 record also shows the impact of millennial scale sea level oscillations during MIS 3, evidencing the occurrence of relative high sea levels during each of the Dansgaard-Oescheger Greenland warm interstadials. Hence, the PRGL1-4 record represents the first evidence of a consistent coupling between sea level oscillations and millennial scale climate variability associated with Dansgaard-Oeschger cycles.
\end{abstract}

Palabras clave: variabilidad climática de escala milenaria, estadio isotópico marino (MIS 3), oscilaciones del nivel del mar, tamaño de grano, Golfo de León.

Key words: millennial scale climate variability, marine isotope stage 3 (MIS 3), sea level oscillations, grain-size, Gulf of Lion.

Enviado el 5 de noviembre de 2012 Aceptado el 4 de enero de 2013

* Correspondencia: GRC en Geociències Marines, Dept. d'Estratigrafia, Paleontologia i Geociències Marines, Universitat de Barcelona. E-mail: jfrigola@ub.edu

\title{
1. Introducción
}

La historia climática del planeta en los últimos 530000 años se ha caracterizado por la sucesión de ciclos glaciales/interglaciales con una periodicidad de 100000 años, modulados a su vez por ciclos de 23000 y 41000 años, todos ellos asociados a los cambios en la insolación producidos por variaciones en la configuración de la órbita terrestre (Imbrie et al., 1992; Duplessy et al., 2005). No obstante, más allá del control que ejercen los forzamientos astronómicos sobre la distribución latitudinal y estacional de la insolación, se desconocen con exactitud los mecanismos del sistema climático que die- 
ron lugar a la formación de los grandes casquetes polares durante las épocas glaciales. Se desconoce también si el volumen de hielo acumulado en los casquetes polares estuvo totalmente controlado por las variaciones de insolación en el hemisferio norte o si estas últimas pudieron generar mecanismos de retroalimentación dentro del sistema climático que determinaron el avance de los hielos (Imbrie et al., 1992; Ruddiman, 2003). Así pues, paralelamente a los ciclos glaciales/interglaciales, se sucedieron oscilaciones del nivel del mar de $120 \mathrm{~m}$ de magnitud en respuesta a los cambios del volumen de hielo acumulado en los mantos polares (Imbrie et al., 1992; Siddall et al., 2006).

Los cambios en el volumen de hielo acumulado en los polos están directamente relacionados con el volumen de agua en los océanos, y es por ello que el estudio de la variabilidad climática de la Tierra ha estado siempre muy ligado al de las oscilaciones globales del nivel del mar. En consecuencia, el conocimiento de los cambios globales del nivel del mar puede ser clave para descifrar el papel que los mantos de hielo polares han jugado en la historia climática del planeta durante el último medio millón de años, tanto a escala orbital como milenaria. Las reconstrucciones del nivel del mar suelen llevarse a cabo mediante dos metodologías. La primera consiste en reconstrucciones discretas a partir de marcadores de la posición del nivel del mar en la costa, habitualmente estructuras y depósitos directamente relacionados con la dinámica costera, como plataformas de abrasión y terrazas coralinas emergidas (Yokoyama et al., 2001; Thompson y Goldstein, 2005; Dabrio et al., 2011). La segunda metodología consiste en reconstrucciones continuas a partir de las oscilaciones de la relación de isótopos de oxígeno $\left({ }^{16} \mathrm{O}\right.$ y $\left.{ }^{18} \mathrm{O}\right)$ medidos en foraminíferos planctónicos y bentónicos (Shackleton et al., 2000; Chappell, 2002; Lea et al., 2002; Waelbroeck et al., 2002; Siddall et al., 2003; Miller et al., 2005; Rohling et al., 2009). Este último método ha permitido obtener reconstrucciones continuas del nivel del mar para los últimos $800 \mathrm{ka}$, en algunos casos con resoluciones cercanas a la escala milenaria, por lo que las mismas han sido ampliamente utilizadas como estándares en cronoestratigrafía marina (Emiliani, 1955; Martinson et al., 1987; Lisiecki y Raymo, 2005). Sin embargo, limitaciones inherentes al método de cálculo del nivel del mar, así como en la datación de los registros, hacen difícil determinar con precisión la amplitud y la edad exacta de los cambios del nivel del mar de escala orbital y milenaria (Siddall et al., 2008).

A escala milenaria, resulta de gran interés conocer con mayor exactitud la amplitud y la edad precisa de las oscilaciones del nivel del mar durante el último período glacial, en el Estadio Isotópico Marino (MIS) 3, para saber qué papel han jugado las variaciones en el volumen de hielo de los mantos polares en la variabilidad climática rápida de la Tierra (Siddall et al., 2008). De hecho, hay una notable controversia en torno al origen de las oscilaciones del nivel del mar observadas durante el MIS 3. Algunos autores sugieren que las subidas del nivel del mar durante este período se deberían a contribuciones del manto de hielo antártico (Siddall et al., 2003, 2008; Rohling et al., 2009), mientras que otros apuntan a aportaciones de los mantos de hielo del hemisferio norte (Arz et al., 2007; Sierro et al., 2009).

Las oscilaciones del nivel del mar, junto con los aportes de sedimento y la subsidencia, han modelado los márgenes continentales pasivos, en los que la migración de las desembocaduras fluviales asociada a las fluctuaciones del nivel del mar ha generado la 
acumulación de secuencias deposicionales regresivas y transgresivas. El estudio sismoestratigráfico de este tipo de secuencias en márgenes continentales con tasas de sedimentación altas y una elevada preservación, ha permitido desarrollar modelos deposicionales basados en las fluctuaciones del nivel del mar (Vail et al., 1977; Posamentier y Vail, 1988). Así pues, el estudio en detalle de registros sedimentarios con un buen control cronoestratigráfico en este tipo de márgenes puede ser una herramienta muy útil para mejorar las reconstrucciones de los cambios del nivel del mar, tanto a escala orbital como milenaria.

En este trabajo presentamos los resultados del estudio del registro sedimentario del talud superior del Golfo de León (GoL) donde a partir de las variaciones de indicadores sedimentológicos como el tamaño de grano y la composición elemental del sedimento, y una robusta cronoestratigrafía, se ha podido redefinir el modelo de sedimentación del margen durante los últimos 530 ka y también analizar a muy alta resolución las oscilaciones del nivel del mar durante el MIS 3.

\section{El margen progradante del Golfo de León: presente y pasado}

El margen pasivo del GoL tiene forma de media luna, con una plataforma continental de $70 \mathrm{~km}$ de anchura máxima que cubre un área de unos $11000 \mathrm{~km}^{2}$, y está incidido por numerosos cañones submarinos que conectan la plataforma continental con la base del talud (Fig. 1). En la plataforma del GoL se distinguen tres regiones fisiográficas bien diferenciadas: la plataforma interna, ocupada por el prisma deltaico y costero actual; la plataforma intermedia, relativamente extensa, plana y cubierta principalmente por arenas relictas; y la plataforma externa, estrecha y de morfología suave, que enlaza con el borde de plataforma y el talud superior (Berné et al., 2004) (Fig. 1). Esta fisiografía resulta en un muy notable espacio de acomodación para los depósitos de origen fluvial cuya principal fuente es el Río Ródano, el más caudaloso y con mayor descarga sólida del Mediterráneo Occidental (Pont et al., 2002; Ludwig et al., 2009). Los sedimentos de origen fluvial, y especialmente los groseros, son atrapados mayoritariamente en la plataforma interna, en el propio delta del Ródano y en el bien desarrollado prisma costero actual. Sin embargo, una fracción de los aportes fluviales es removilizada y transportada hacia la plataforma intermedia y externa gracias a procesos de erosión y transporte como las grandes tormentas y los flujos asociados a la formación y a las cascadas de aguas densas de plataforma (DSWC). Estos procesos han sido descritos en las condiciones actuales como los más efectivos en el transporte de sedimentos desde la plataforma hacia la cuenca a través principalmente de los cañones submarinos (Ulses et al., 2005; Bassetti et al., 2006; Canals et al., 2006; Palanques et al., 2006; Dufois et al., 2008; Sanchez-Vidal et al., 2008, 2012; Pasqual et al., 2010). La Corriente Liguro-Provenzal o Corriente del Norte (NC), que circula paralela al talud en dirección SW, puede también afectar al transporte y acumulación de sedimentos finos en la plataforma y el talud, contribuyendo así a la sedimentación de fondo (background sedimentation) (Amblas et al., 2012).

A escala glacial/interglacial, las fluctuaciones del nivel del mar del Cuaternario han modelado la plataforma del GoL (Berné et al., 2004), proporcionando un gran espacio de 


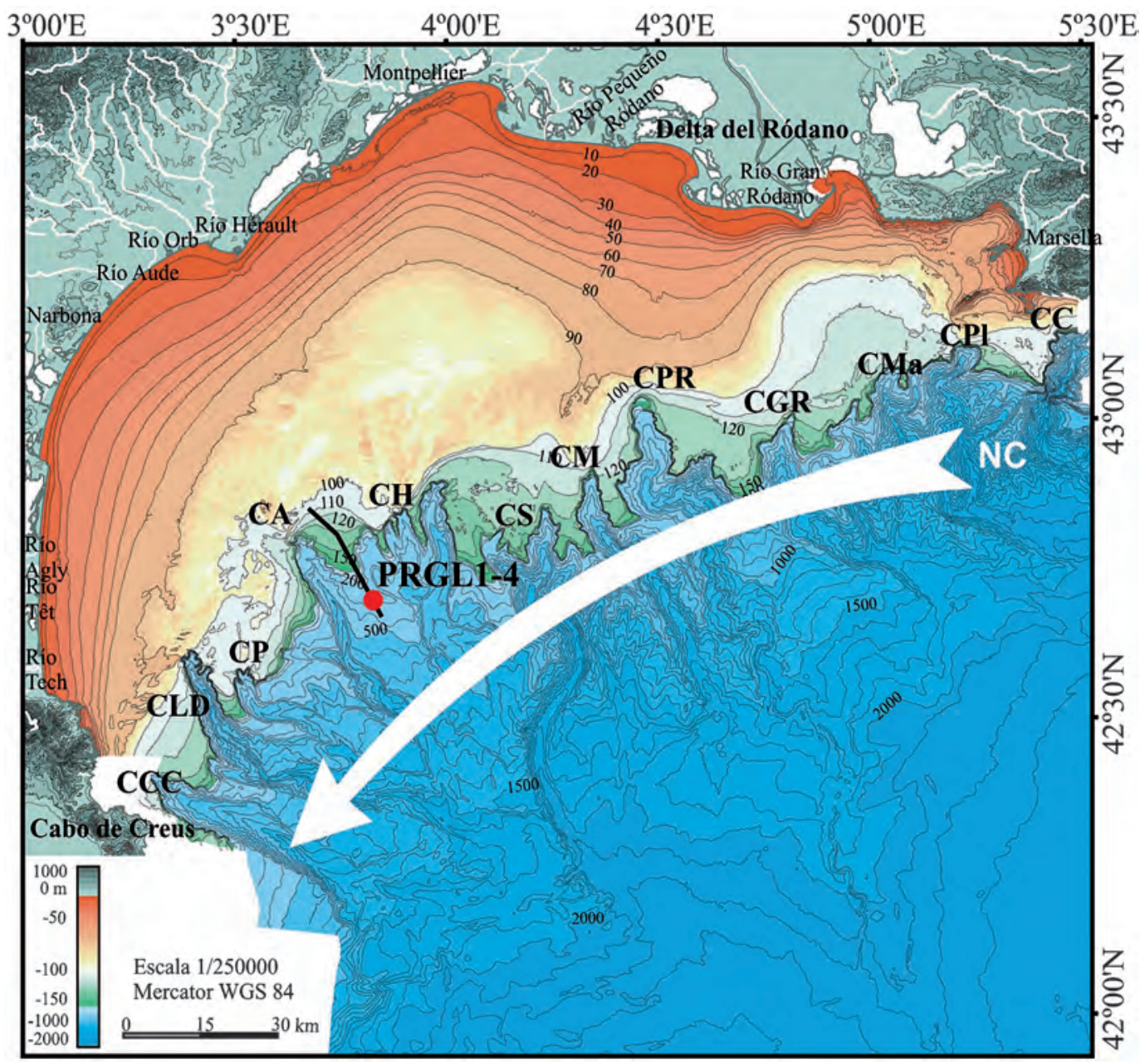

Figura 1. Mapa batimétrico del Golfo del León (modificado de Berné et al., 2004). La escala de colores permite identificar los tres dominios de plataforma descritos en el texto:

1) la plataforma interna, entre 0 y $90 \mathrm{~m}$ de profundidad, que se caracteriza por una pendiente regular correspondiente al prisma deltaico moderno, con colores rojizos y anaranjados;

2) la plataforma intermedia, entre 90 y 110-120 m de profundidad, que es muy plana y presenta una morfología irregular, en colores amarillentos; y 3) la plataforma externa, entre 110-120 m de profundidad y el borde de plataforma, en colores grises y verdes. Los colores azulados representan el talud y el glacis continental. El punto rojo marca la localización del sondeo PRGL1-4, mientras que la línea que cruza en dirección NW-SE ilustra el perfil sísmico mostrado en la Fig. 2A. Numerosos cañones submarinos conectan la plataforma con el pie del talud y la cuenca profunda. CCC: Cañón del Cap de Creus; CLD: Cañón de Lacaze-Duthiers;

CA: Cañón del Aude; CH: Cañón del Hérault; CS: Cañón de Sète; CM: Cañón de Martí; CPR: Cañón del Pequeño Ródano; CGR: Cañón del Gran Ródano; CMa: Cañón de Marsella;

CPl: Cañón del Planier; CC: Cañón de Cassidaigne. La Corriente del Norte (NC) circula paralela a la costa en dirección $S W$ y a veces penetra en la plataforma (flecha blanca). 
acomodación para el agua y la acumulación de sedimentos durante los períodos de nivel del mar alto como el actual (highstands), mientras que durante los períodos de nivel del mar bajo (lowstands) la plataforma quedó parcial o totalmente expuesta a condiciones subaéreas. Estas circunstancias dieron lugar a la acumulación de secuencias regresivas y transgresivas en el talud superior durante los últimos $530 \mathrm{ka}$ (Fig. 2A). Las elevadas tasas de subsidencia del margen, $250 \mathrm{mMa}^{-1}$ en el eje de la plataforma continental (Rabineau, 2001), han facilitado la preservación de dichas secuencias puesto que se mantuvieron siempre sumergidas. Estas secuencias, predominantemente regresivas, se conocen con el nombre de Unidades Regresivas Progradantes (Regressive Progradational Units, RPU) y confieren al margen un carácter regresivo forzado (Tesson et al., 1990, 2000; Posamentier et al., 1992). Trabajos previos, basados principalmente en la interpretación de perfiles de sísmica de reflexión y de registros sedimentarios cortos, permitieron en una primera interpretación identificar cinco RPUs que fueron consideradas el resultado de las oscilaciones del nivel del mar de 100 ka de ciclicidad (Rabineau et al., 1998, 2005; Bassetti et al., 2008). Sin embargo, para establecer con mayor certeza el origen de tales secuencias, y en concreto la contribución de las oscilaciones del nivel del mar de los ciclos de 100 ka y/o de 23 ka, faltaba datar con mayor precisión los límites de secuencia de las RPUs (Lobo et al., 2004).

\section{Material y métodos}

Este trabajo se basa en análisis llevados a cabo en el sondeo PRGL1-4, de $300 \mathrm{~m}$ de longitud, recuperado en el talud superior del GoL a $298 \mathrm{~m}$ de profundidad $\left(42^{\circ}\right.$ $41.39^{\prime} \mathrm{N}, 03^{\circ} 50.26^{\prime} \mathrm{E}$ ), en el interfluvio entre los cañones submarinos del Aude y del Hérault durante la campaña oceanográfica PROMESS1 a bordo del barco ruso V/M Bavenit operado por la compañía holandesa FUGRO Engineers B.V. La parte superficial del registro también ha sido estudiada en el testigo IMAGES MD99-2348, de $22.77 \mathrm{~m}$ de longitud, recuperado en la misma posición (Fig. 1 y 2A).

Los análisis granulométricos se llevaron a cabo, a intervalos de $20 \mathrm{~cm}$, en la fracción total y en la fracción des-carbonatada con un analizador láser Coulter LS100. La materia orgánica fue eliminada con agua oxigenada en exceso mientras que los carbonatos de la fracción des-carbonatada fueron eliminados con ácido clorhídrico $1 \mathrm{M}$. Se ha utilizado la relación limo/arcilla (silt/clay ratio) de la fracción des-carbonatada como indicador de la energía del medio en el momento de la deposición (Frigola et al., 2007).

El análisis elemental (Ca, Ti, K y Fe) del sedimento se efectuó, con carácter no destructivo, a una resolución de $4 \mathrm{~cm}$ mediante un escáner de testigos por fluorescencia de rayos X (XRF core scanner) Avaatech de la primera generación en la Universidad de Bremen. La buena correlación existente entre el perfil de $\mathrm{Ca}$ y el contenido de arcillas y las tasas de sedimentación sugieren que, en el lugar de estudio, el Ca es un indicador de aportes fluviales. En la actualidad, la calcita es el principal componente mineralógico de las partículas en suspensión aportadas por el Río Ródano (Pont et al., 2002), lo cual apoya esta interpretación. 


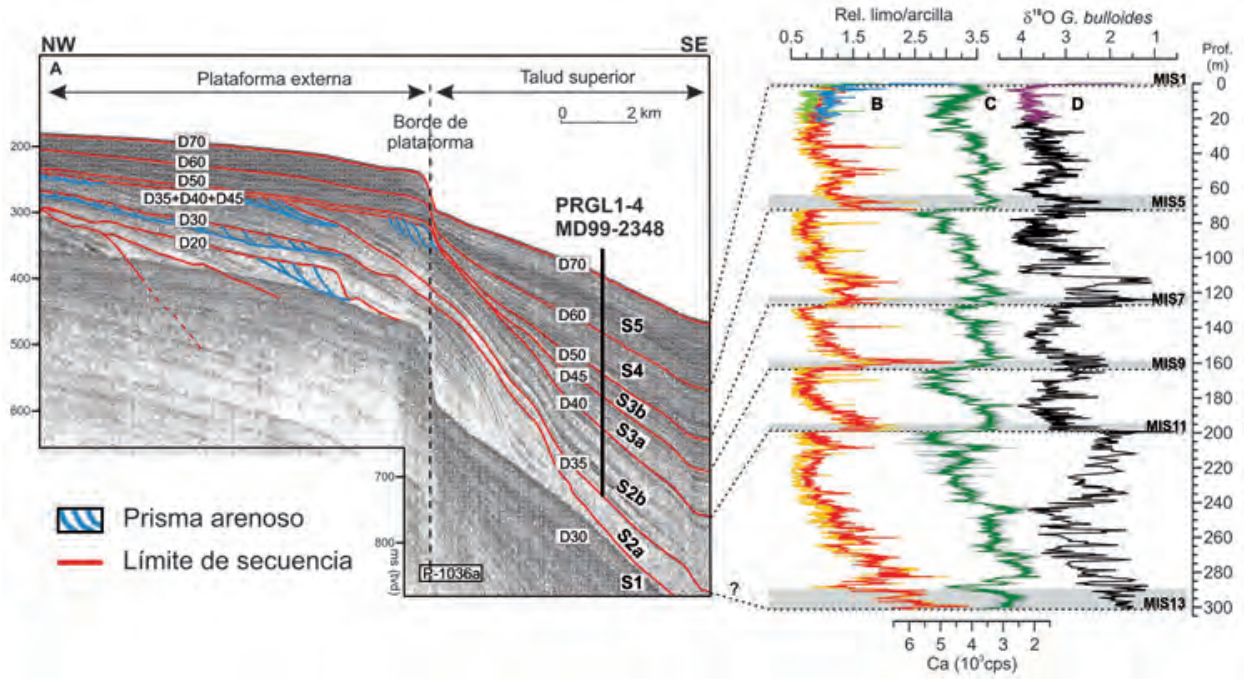

Figura 2. (A) Perfil de sísmica de reflexión a través de la plataforma externa y el talud superior en el interfluvio entre los cañones del Aude y del Hérault en el que se muestra la localización del sondeo PRGL1-4 y del testigo MD99-2348 (modificado de Jouet, 2007).

En el perfil se ilustran las secuencias sismoestratigráficas (S5-S1) correspondientes a las RPUs en el talud superior y los límites de secuencia (D70-D30). Las líneas azules muestran sistemas deltaicos progradantes depositados durante períodos glaciales pretéritos.

Registros del testigo MD99-2348 y del sondeo PRGL1-4 de la (B) relación limo/arcilla, (C) el contenido de Ca, y (D) el $\delta^{18} \mathrm{O}$ de G. bulloides.

El modelo de edad para los últimos 100 ka se obtuvo por sincronización de los registros de $\delta^{18} \mathrm{O}$ de $G$. bulloides y de abundancia de foraminíferos planctónicos templados-cálidos (Sierro et al., 2009) con el registro de $\delta^{18} \mathrm{O}$ del testigo de hielo NGRIP (NGRIP, 2004; Andersen et al., 2006; Svensson et al., 2008). Entre 100 y $530 \mathrm{ka}$ el modelo de edad se obtuvo por sincronización del registro de $\delta^{18} \mathrm{O}$ del sondeo PRGL1-4 con el registro de isótopos bentónicos LR04 (Lisiecki y Raymo, 2005) (Frigola et al., 2012). La resolución temporal derivada del modelo de edad oscila entre los 160 y los 1550 años durante los períodos glaciales y los interglaciales, respectivamente.

\section{Resultados y discusión}

\subsection{Oscilaciones del nivel del mar a escala glacial/interglacial}

Los registros de la relación limo/arcilla y del Ca en el sondeo PRGL1-4 muestran una secuencia compuesta por cinco unidades. Los registros tienen forma de dientes de sierra que, para cada unidad, muestran una tendencia a la disminución del tamaño de grano y al incremento en el contenido de Ca hacia techo, acabando con un incremento muy abrupto de la relación limo/arcilla y una disminución rápida en el contenido de Ca 
(Fig. 2B y C). El final abrupto de las unidades sedimentarias se correlaciona con los reflectores principales de los perfiles de sísmica de reflexión de alta resolución que pasan por el punto de muestreo (Fig. 2A, B y C), lo que sugiere que dichos reflectores son la expresión sismoestratigráfica de los límites de las unidades sedimentarias. Esta observación, junto con la cronoestratigrafía basada en el registro de $\delta^{18} \mathrm{O}$ de G. bulloides (Sierro et al., 2009) (Fig. 2D), revela que cada una de estas unidades corresponde a un episodio regresivo de bajada del nivel del mar durante un ciclo interglacial/glacial de $100 \mathrm{ka}$ (Frigola et al., 2012). Se ha podido interpretar así el modelo deposicional del talud superior del GoL, donde se han identificado siete unidades (S1, S2a, S2b, S3a, S3b, S4 y S5) (Jouet, 2007) (Fig. 2A) en vez de las cinco previamente definidas a través de perfiles de sísmica de reflexión (Rabineau, 2001). El sondeo PRGL1-4 no alcanzó a perforar las unidades más profundas $\mathrm{S} 1$ y $\mathrm{S} 2 \mathrm{a}$, y según el registro de $\delta^{18} \mathrm{O}$ de $G$. bulloides se estima que la base del registro se sitúa en torno a los 530 ka, en el MIS 13 (Fig. 2D). En consecuencia, las cinco unidades perforadas y descritas del sondeo PRGL1-4 corresponden a las RPUs depositadas durante las oscilaciones globales del nivel del mar de los últimos cinco ciclos glaciales. El incremento abrupto de la relación limo/arcilla y la rápida disminución en el contenido de $\mathrm{Ca}$ al final de cada unidad son debidos a la rápida subida del nivel del mar durante las transiciones glacial/interglacial que dieron lugar a la inundación de la plataforma y a la migración subsiguiente hacia tierra de los sistemas deltaicos y del prisma litoral. Este drástico cambio en las condiciones de sedimentación en el talud superior generó los límites de unidad definidos por los resultados analíticos.

La deposición de cada RPU en el talud superior del GoL resulta de la migración de los sistemas fluviales hacia el borde de la plataforma, con el consiguiente incremento de los aportes fluviales asociado a la bajada del nivel del mar en cada ciclo de $100 \mathrm{ka}$. Por esta razón las tasas de sedimentación más altas en el talud superior (1.5-2.5 mka $\mathrm{ma}^{-1}$ ocurrieron durante los períodos en que la distancia a las desembocaduras de los ríos era menor, es decir, durante los niveles del mar más bajos (lowstands) de los períodos glaciales (Fig. $3 \mathrm{~F}$ y $4 \mathrm{~A}$ ). El incremento de las tasas de sedimentación ligado a la bajada del nivel del mar a lo largo de un período glacial está particularmente bien resuelto para el último período glacial (MIS 2, 3 y 4) gracias a la robustez del modelo de edad (Fig. 4F). Además, la sincronía entre los valores más bajos de la relación limo/arcilla y los más altos en el contenido de Ca durante los lowstands glaciales confirma el incremento concomitante de los aportes de finos fluviales en los interfluvios del talud superior (Fig. 3C y D). En cambio, las tasas de sedimentación en el talud superior del GoL fueron mínimas (0.10-0.25 m kyr $\left.{ }^{-1}\right)$ durante los niveles del mar altos (highstands) de los períodos interglaciales, debido a la migración hacia tierra de los sistemas fluviales (Fig. 3F y 4B), tal y como ilustra el prisma epicontinental moderno (Fig. 1). El gran contraste entre las tasas de sedimentación en una y otra situación ha dado lugar a un registro en acordeón, expandido durante los períodos glaciales, de lo que resulta una resolución temporal elevada, y comprimido durante los períodos interglaciales, cuya representación en el registro está minimizada. En consecuencia, cada subida del nivel del mar supuso una fuerte caída de las tasas de sedimentación en el talud superior y, por lo tanto, una pérdida de resolución temporal, limitación que afecta también a la datación precisa de los eventos rápidos. De hecho, las bajas tasas de sedimentación en el talud superior durante largos intervalos de tiempo en los períodos inter- 


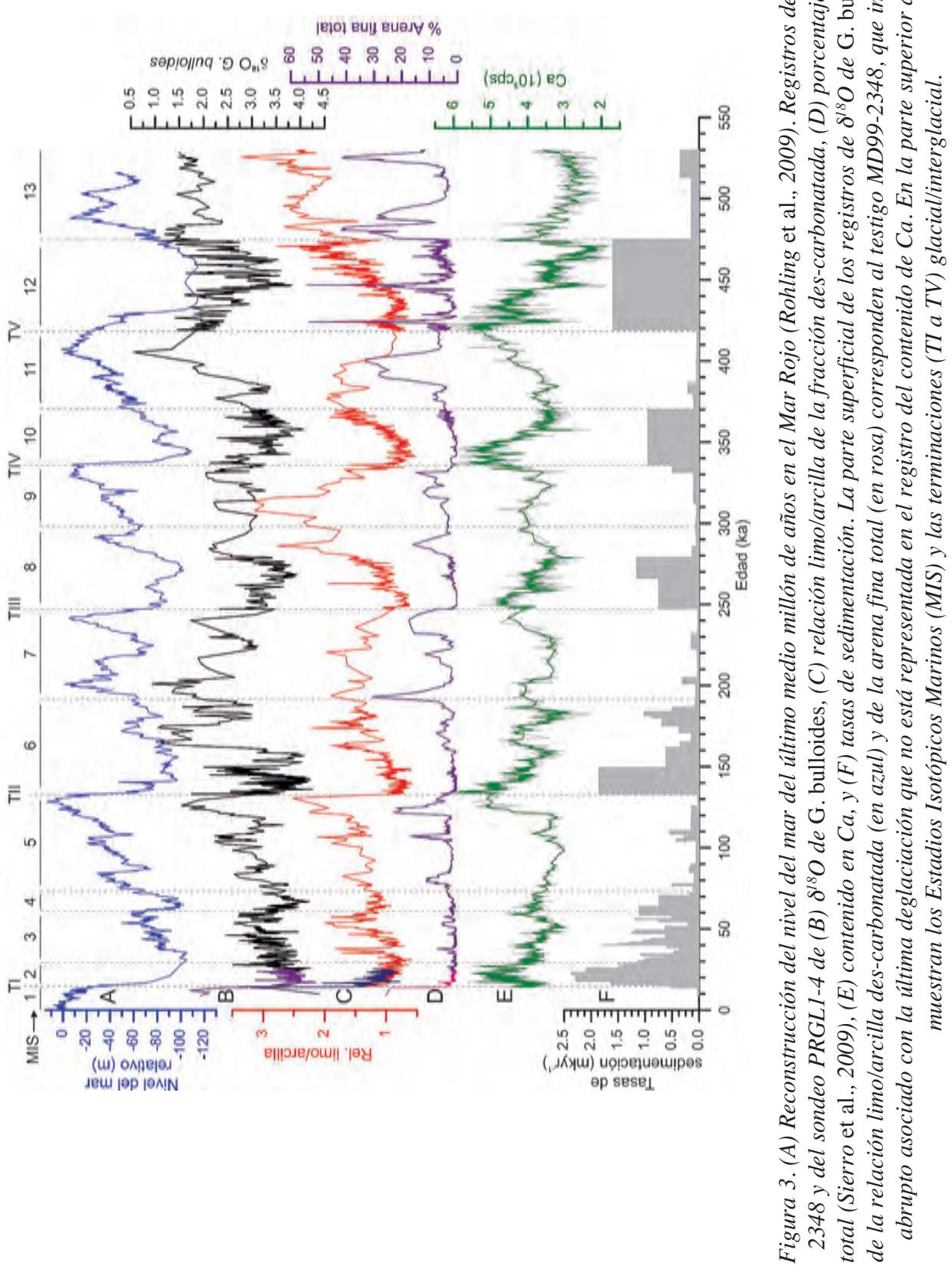


glaciales determinaron la formación de capas condensadas (Condensed Layers, CL), capas ricas en arenas de foraminíferos, según muestran los incrementos en el registro de arenas finas de la fracción total (Sierro et al., 2009) (Fig. 3D).

Estos resultados pueden parecer contradictorios, ya que a priori podría pensarse que lo más lógico sería encontrar que las partículas más gruesas se hubiesen acumulado en el talud superior cuando los puntos de entrada de sedimentos fluviales estuviesen más próximos, es decir, durante los períodos glaciales con nivel del mar bajo, y viceversa. La abundante acumulación de finos durante los períodos glaciales se puede explicar precisamente por las voluminosas aportaciones de arcillas procedentes de los sistemas deltaicos cercanos en los interfluvios, tal y como lo demuestra los elevados contenidos de $\mathrm{Ca}$, mayoritariamente de origen fluvial, y las altas tasas de sedimentación registradas durante estos intervalos (Fig. 3E y F). El registro granulométrico del sondeo PRGL1-4 también muestra que los sedimentos más gruesos se acumularon durante los interglaciales, cuando la línea de costa y los sistemas deltaicos estaban lejos del talud superior. Este incremento del tamaño de grano durante los períodos interglaciales con tasas de sedimentación bajas no era debido únicamente a las CLs, sino también a una mayor aportación de partículas terrígenas, como demuestran los incrementos en la relación limo/arcilla de la fracción des-carbonatada (Fig. 3C). Estos resultados sugieren que la inundación de la plataforma del GoL durante los interglaciales habría re-activado procesos hidrosedimentarios de alta energía capaces de transportar partículas gruesas hasta el talud superior, como sucede en la actualidad durante los temporales y los episodios de DSWC (Bassetti et al., 2006; Canals et al., 2006; Dufois et al., 2008). Así pues, la inundación de la plataforma durante los ascensos del nivel del mar habría proporcionado, por un lado, un espacio de acomodación suficiente para mantener los sistemas deltaicos alejados del talud superior, provocando así una disminución de las tasas de sedimentación y, por otro lado, habría reactivado procesos erosivos y de transporte asociados al efecto de los forzamientos atmosféricos sobre la delgada capa de agua de la plataforma. Sin embargo, cuando la plataforma estaba expuesta, durante los períodos glaciales, estos procesos o no existían, como en el caso de las DSWC, que necesitan una extensa plataforma sobre la que puedan enfriarse y evaporarse las aguas, o su intensidad y efectos eran menores, por lo que el aporte de sedimentos al talud superior pasaba a depender esencialmente de la proximidad de las desembocaduras fluviales. Durante los períodos de transición el volumen de agua que participaba en las DSWC y en otros procesos erosivos, así como la propia superficie inundada de la plataforma, eran menores, por lo que cabría esperar que el transporte de material grueso hacia el talud fuese también inferior. Por esta razón, los cambios en la relación limo/arcilla también responderían al área de la plataforma inundada, lo cual explicaría la buena correlación entre el registro de este índice con las oscilaciones del nivel del mar en los últimos $530 \mathrm{ka}$ (Fig. 3A y $\mathrm{C})$, hecho particularmente evidente durante el último ciclo glacial, en el que se dispone de un mayor control cronoestratigráfico. Estos resultados sugieren que el desarrollo sedimentario del GoL durante los últimos 530 ka está modulado por la combinación de dos procesos: (1) las fluctuaciones en el aporte de sedimento debidas a la migración de los sistemas fluviales, y (2) la activación y desactivación de procesos erosivos y de transporte propios de la plataforma continental (e.g. DSWC), controlados ambos por las oscilaciones globales del nivel del mar (Fig. 4) (Frigola et al., 2012). 


\section{A) Lowstand}

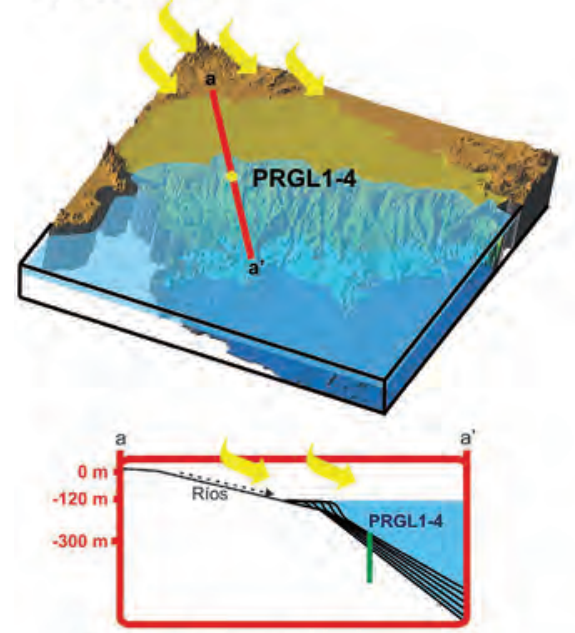

- Plataforma emergida

- Sistemas deltaicos en el borde de plataforma

- Incremento de la acumulación de finos en el talud superior.

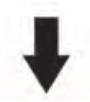

\section{DSWC Reducido o inexistente}

\section{B) Highstand}
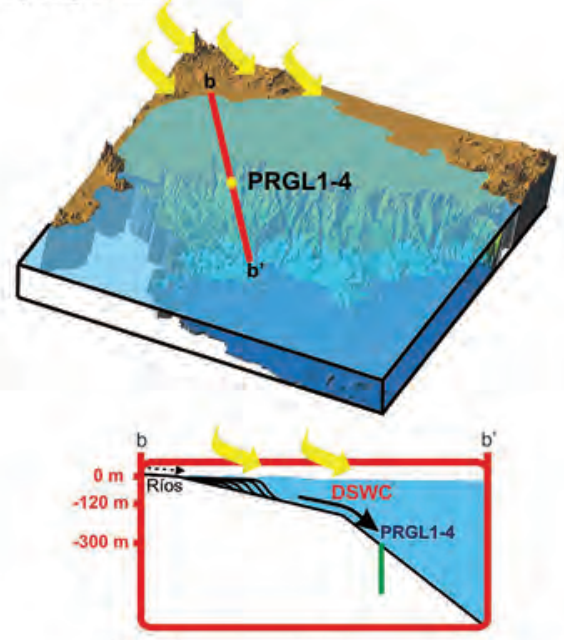

- Plataforma inundada

- Sistemas deltaicos en la plataforma interna

- Disminución de la sedimentación fluvial en el talud superior.

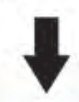

\section{Activación DSWC}

Figura 4. Esquema conceptual del modelo de sedimentación de escala orbital en el Golfo de León. (A) Durante las condiciones del nivel del mar bajo, lowstands, la plataforma continental queda emergida y los sistemas deltaicos migran hacia el borde de plataforma y el talud superior, donde se incrementan las tasas de sedimentación de finos. (B) La inundación de la plataforma con la subida del nivel del mar durante los períodos interglaciales empuja y atrapa los sistemas deltaicos en la plataforma interna, lo cual provoca una disminución del aporte de material fluvial al talud superior. La creación de una lámina de agua sobre la plataforma facilita la reactivación de procesos erosivos como el DSWC, capaces de transportar partículas gruesas hacia el talud superior. Las flechas amarillas representan los vientos del $N$ y el NW que soplan con fuerza y persistencia en esta zona, y son los causantes de la densificación y el hundimiento de las aguas superficiales.

\subsection{Variaciones del nivel del mar durante el MIS 3}

Teniendo en cuenta el modelo deposicional descrito, las elevadas tasas de sedimentación, entre 0.2-2 $\mathrm{m} \mathrm{ka}^{-1}$, y el exhaustivo control cronoestratigráfico del último período glacial (Fig. 5A y B), las variaciones en la relación limo/arcilla del sondeo PRGL1-4 pueden servir para evaluar las oscilaciones del nivel del mar de escala milenaria durante el MIS 3 en el contexto de la variabilidad climática rápida de los ciclos de DansgaardOeschger (DO) descrita para este período (Dansgaard et al., 1984; Voelker, 2002). De hecho, ninguna reconstrucción del nivel del mar ha sido capaz de resolver fluctuaciones 


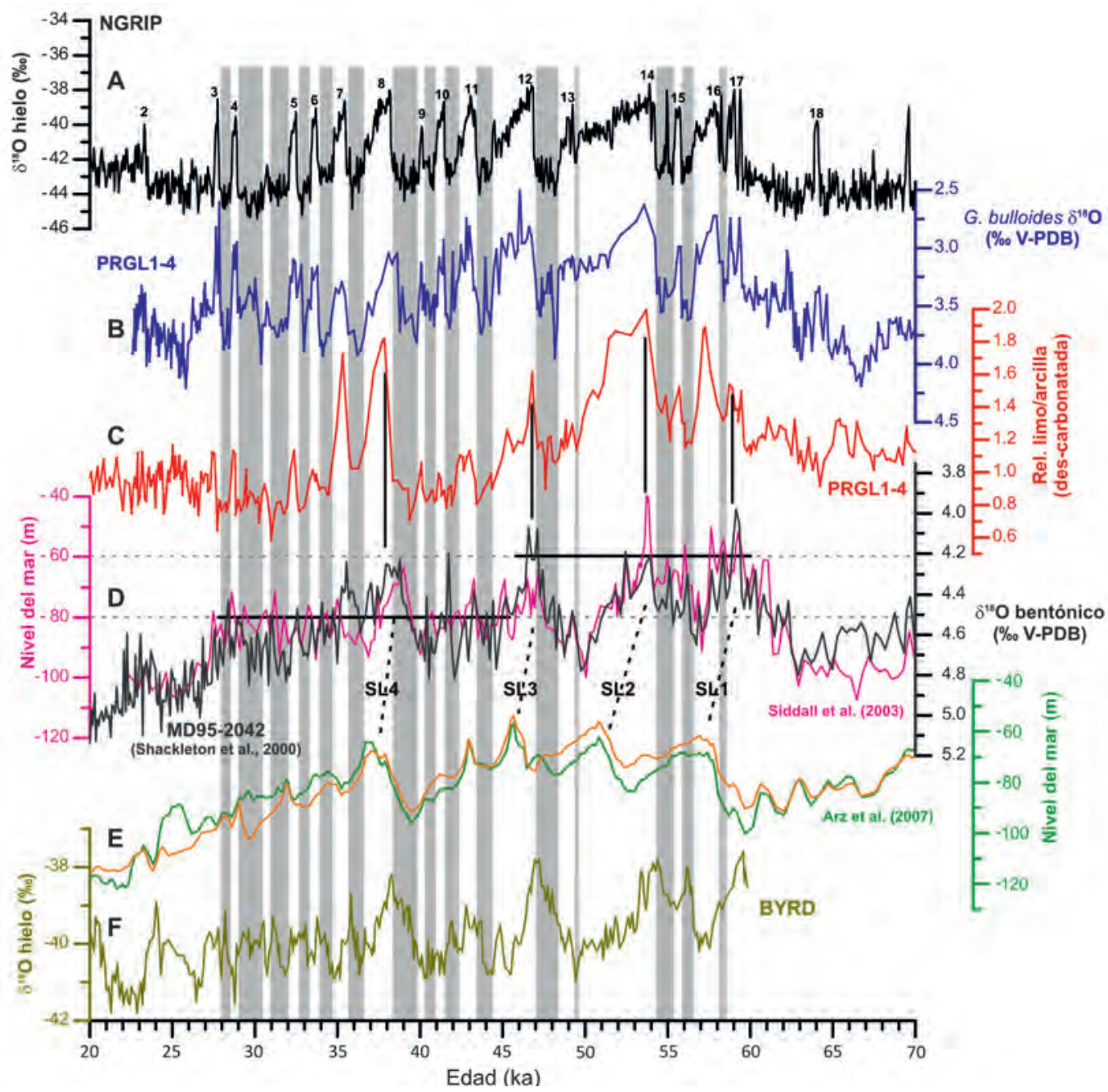

Figura 5. Comparación de diferentes registros de variabilidad climática y reconstrucciones del nivel del mar para el MIS 3, todos ellos representados con el modelo de edad del testigo de hielo de Groenlandia NGRIP (Svensson et al., 2008)(A). (B) Registro de $\delta^{18} \mathrm{O}$ de G. bulloides (en azul) y $(C)$ de la relación limo/arcilla (en rojo) del sondeo PRGL1-4. (D) Registro de $\delta^{18} O$ bentónico del testigo MD95-2042 del margen de Portugal (Shackleton et al., 2000) (en gris), comparado con la reconstrucción del nivel del mar obtenida en el Mar Rojo (en rosa) (Siddall et al., 2003), mostrando con líneas horizontales que el nivel del mar durante el inicio del MIS 3 era unos 20 m más alto que al final. Las líneas discontinuas muestran cuatro niveles del mar altos durante el MIS 3 (SL1, 2, 3 y 4). (E) Reconstrucción del nivel del mar del Mar Rojo basada en dos correcciones diferentes de temperatura (Arz et al., 2007). (F) Registro de $\delta^{18} \mathrm{O}$ del testigo de hielo de la Antártida BYRD (Blunier y Brook, 2001) sincronizado con el testigo NGRIP de Groenlandia a través de sus curvas de metano (Svensson et al., 2008). Los números sobre la curva de NGRIP señalan los interestadiales cálidos, mientras que las barras grises muestran los estadiales fríos de los ciclos de Dansgaard-Oeschger. 
de menos de $12 \mathrm{~m}$ de amplitud durante períodos cortos, en torno a 1 ka (Siddall et al., 2008). Sin embargo, grandes desestabilizaciones de los mantos de hielo durante los estadios fríos de los ciclos de DO (Greenland stadials, GS) sugieren que el nivel del mar pudo haber oscilado en cada uno de ellos (Bond y Lotti, 1995; van Kreveld et al., 2000). Una revisión exhaustiva de las reconstrucciones del nivel del mar durante el MIS 3 destacó dos hechos relevantes en común: 1) el nivel del mar era aproximadamente $20 \mathrm{~m}$ superior al inicio que al final del MIS 3; y 2) se produjeron cuatro oscilaciones del nivel del mar de 20-30 m de amplitud (Fig. 5D) (Siddall et al., 2008). El registro granulométrico del sondeo PRGL1-4 corrobora estas dos observaciones, lo cual demuestra la gran sensibilidad de este registro ante las fluctuaciones del nivel del mar durante dicho período (Fig. 5C). De hecho, la relación limo/arcilla muestra una serie de incrementos del tamaño de grano que sugieren la existencia de oscilaciones del nivel del mar de escala milenaria a lo largo del MIS 3 (Fig. 5C). Incrementos puntuales del nivel mar habrían contribuido a reducir el aporte de finos al talud superior, a la vez que habrían incrementado el volumen de agua sobre la plataforma expuesto a forzamientos atmosféricos, favoreciendo así procesos como el DSWC que, habrían reforzado el transporte de partículas más gruesas al talud superior. La sincronía entre los registros de $\delta^{18} \mathrm{O}$ de $G$. bulloides y la relación limo/arcilla del sondeo PRGL1-4 confirma la ocurrencia de niveles del mar altos durante cada uno de los interestadios cálidos de los ciclos de DO (Greenland interstadials, GIS) (Fig. 5A y C). Esta es la primera evidencia de un acoplamiento consistente, pico a pico, entre las oscilaciones del nivel del mar y la variabilidad climática de escala milenaria durante el MIS 3 (Frigola et al., 2012).

De la correlación de los niveles del mar altos con los GIS podría interpretarse que la aportación principal al volumen de agua responsable de tales ascensos fueron los mantos polares del Atlántico Norte, como sugieren otros registros (Arz et al., 2007; Sierro et al., 2009). Sin embargo, la ocurrencia de los niveles del mar altos en la fase inicial de los GIS se ha observado también en reconstrucciones del nivel del mar que han sido correlacionadas con la variabilidad climática de la Antártida (Shackleton et al., 2000; Siddall et al., 2003) (e.g. GIS 14, 12 y 8) (Fig. 5D). Si bien el registro del sondeo PRGL1-4 muestra oscilaciones del nivel del mar de escala milenaria durante cada uno de los ciclos de DO, el mismo presenta un par de limitaciones importantes que no permiten clarificar el origen de los ascensos del nivel del mar citados. La primera es que las variaciones en la relación limo/arcilla no se pueden correlacionar directamente con las fluctuaciones del nivel del mar, por lo que no es posible establecer la amplitud de los ascensos del nivel del mar de cada ciclo de DO. La segunda limitación está relacionada con la propia subida del nivel del mar y la caída subsiguiente de las tasas de sedimentación en el talud superior, la cual resulta en una pérdida de la resolución temporal del registro que imposibilita establecer con exactitud el inicio de los ascensos del nivel del mar, los cuales pudieron producirse tanto al inicio de los GIS como durante los GS.

\section{Conclusiones}

El registro sedimentario del Pleistoceno en el Golfo de León está formado por el apilamiento de RPUs, de las cuales las correspondientes a los últimos cinco ciclos glaciales han sido perforadas con el sondeo PRGL1-4, de 300 m de longitud, en el talud superior. 
La formación de estas unidades ha estado controlada por las oscilaciones globales del nivel del mar de escala orbital del ciclo de $100 \mathrm{ka}$. La buena correlación existente entre el registro granulométrico del sondeo y los perfiles de sísmica de reflexión, junto con la robusta cronoestratigrafía de que se ha dispuesto, han permitido reinterpretar la naturaleza de tales unidades regresivas, a la vez que también han servido para establecer los límites de las secuencias sismoestratigráficas y su correlación con las oscilaciones globales del nivel del mar. Estos resultados muestran el gran potencial del estudio integrado de registros sedimentarios largos en márgenes pasivos para mejorar el conocimiento sobre el efecto de las oscilaciones globales del nivel del mar en su construcción.

Además de provocar la migración de la línea de costa y de los sistemas fluviales hacia el continente, desconectando así el talud superior del aporte directo de sedimentos, los ascensos del nivel del mar tienen la capacidad de reactivar procesos hidrosedimentarios de alta energía, como el DSWC, capaces de erosionar y transportar cantidades significativas de sedimento desde la plataforma interna hasta el talud y la cuenca profunda, modulando así también la sedimentación en la plataforma durante los períodos de nivel del mar alto.

Finalmente, la buena correlación existente entre el registro de limo/arcilla del sondeo PRGL1-4 y las reconstrucciones del nivel del mar durante el MIS 3, junto con la buena cronoestratigrafía proporcionada por el registro de $\delta^{18} \mathrm{O}$ de $G$. bulloides, permiten inferir la ocurrencia de oscilaciones del nivel del mar de escala milenaria en sincronía con los ciclos de DO, registrándose niveles del mar relativamente altos en cada estadio cálido GIS, incluso en los menos intensos. Estos resultados constituyen la primera evidencia del acoplamiento recurrente de las oscilaciones del nivel del mar y la variabilidad climática rápida durante el MIS 3, aunque limitaciones en el propio registro PRGL1-4 impiden determinar la amplitud y datar con exactitud los ascensos del nivel del mar.

\section{Agradecimientos}

Este trabajo ha sido financiado por los proyectos europeos PROMESS1 (EVR1-CT200240024) y HERMIONE (226354-HERMIONE), los proyectos nacionales GRACCIE CONSOLIDER (CSD2007-00067) y DOS MARES (CTM2010-21810-C03-01), y la acción complementaria CGL2005-24147-E. El testigo MD99-2348 se obtuvo en el marco del programa IMAGES. Estamos especialmente agradecidos a los participantes de la campaña PROMESS1 y al personal de los diferentes laboratorios en los que se han tratado las muestras de sedimento. La Generalitat de Catalunya reconoce al GRC en Geociències Marines a través de su programa para grupos de investigación de excelencia (ref. 2009 SGR 1305).

\section{Referencias bibliográficas}

Amblas, D., Gerber, T.P., De Mol, B., Urgeles, R., García-Castellanos, D., Canals, M., Pratson, L.F., Robb, N. Canning, J. 2012. Survival of a submarine canyon during long-term outbuilding of a continental margin. Geology 40, 543-546. 
Andersen, K.K., Svensson, A., Johnsen, S.J., Rasmussen, S.O., Bigler, M., Rothlisberger, R., Ruth, U., Siggaard-Andersen, M.L., Peder Steffensen, J., Dahl-Jensen, D., Vinther, B.M., Clausen, H.B. 2006. The Greenland Ice Core Chronology 2005, 15-42 ka. Part 1: constructing the time scale. Quaternary Science Reviews 25, 3246-3257.

Arz, H.W., Lamy, F., Ganopolski, A., Nowaczyk, N., Patzold, J. 2007. Dominant Northern Hemisphere climate control over millennial-scale glacial sea-level varia-bility. Quaternary Science Reviews 26, 312-321.

Bassetti, M.A., Jouet, G., Dufois, F., Berne, S., Rabineau, M., Taviani, M. 2006. Sand bodies at the shelf edge in the Gulf of Lions (Western Mediterranean): Deglacial history and modern processes. Marine Geology 234, 93-109.

Bassetti, M.A., Berné, S., Jouet, G., Taviani, M., Dennielou, B., Flores, J.A., Gaillot, A., Gelfort, R., Lafuerza, S., Sultan, N. 2008. The 100-ka and rapid sea level changes recorded by prograding shelf sand bodies in the Gulf of Lions (western Mediterranean Sea). Geochemistry Geophysics Geosystems 9, Q11R05. Doi: 10.1029/2007GC001854.

Berné, S., Carré, B., Loubrieu, B., Mazé, J.P., Morvan, L., Normand, A. 2004. Notice de la carte morpho-bathymétrique du Golfe du Lion. Ifremer, Brest.

Blunier, T., Brook, E.J. 2001. Timing of Millennial-Scale Climate Change in Antarctica and Greenland During the Last Glacial Period. Science 291, 109-112.

Bond, G., Lotti, R. 1995. Iceberg discharges into the North Atlantic on millennial time scales during the last deglaciation. Science 267, 1005-1010.

Canals, M., Puig, P., De Madron, X.D., Heussner, S., Palanques, A., Fabres, J. 2006. Flushing submarine canyons. Nature 444, 354-357.

Chappell, J. 2002. Sea level changes forced ice breakouts in the Last Glacial cycle: new results from coral terraces. Quaternary Science Reviews 21, 1229-1240.

Dabrio, C.J., Zazo, C., Cabero, A., Goy, J.L., Bardají, T., Hillaire-Marcel, C., González-Delgado, J.A., Lario, J., Silva, P.G., Borja, F., García-Blázquez, A.M. 2011. Millennial/submillennialscale sea-level fluctuations in western Mediterranean during the second highstand of MIS 5e. Quaternary Science Reviews 30, 335-346.

Dansgaard, W., Johnsen, S.J., Clausen, H.B., Dahl-Jensen, D., Gundestrup, N.S., Hammer, C.U., Oeschger, H. 1984. North Atlantic climatic oscillations revealed by deep Greenland ice cores. En Climate Processes and Climate Sensitivity, J.E. Hansen, T. Takahashi, T. (eds.), American Geophysical Union, Washington, pp. 288-298.

Dufois, F., Garreau, P., Le Hir, P., Forget, P. 2008. Wave- and current-induced bottom shear stress distribution in the Gulf of Lions. Continental Shelf Research 28, 1920-1934.

Duplessy, J.C., Cortijo, E., Masson-Delmotte, V., Paillard, D. 2005. Reconstructing the variability of the climate system: Facts and theories. Comptes Rendus Geoscience 337, 888-896.

Emiliani, C. 1955. Pleistocene temperatures. Journal of Geology 63, 538-578.

Frigola, J., Moreno, A., Cacho, I., Canals, M., Sierro, F.J., Flores, J.A., Grimalt, J.O., Hodell, D. A., Curtis, J.H. 2007. Holocene climate variability in the western Mediterranean region from a deepwater sediment record. Paleoceanography 22, PA2209. Doi:10.1029/2006PA001307.

Frigola, J., Canals, M., Cacho, I., Moreno, A., Sierro, F.J., Flores, J.A., Berné, S., Jouet, G., Dennielou, B., Herrera, G., Pasqual, C., Grimalt, J.O., Galavazi, M., Schneider, R. 2012. A $500 \mathrm{kyr}$ record of global sea level oscillations in the Gulf of Lion, Mediterranean Sea: new insights into MIS 3 sea level variability. Climate of the Past 8, 1067-1077. Doi:10.5194/ cp-8-1067-2012.

Imbrie, J., Boyle, E., Clemens, C., Duffy, A., Howard, W., Kukla, G., Kutzbach, J.E., Martinson, D.G., McIntyre, A., Mix, A.C., Molfino, B., Morley, J.J., Peterson, L.C., Pisias, N.G., Prell, W., Raymo, M.E., Shackleton, N.J., Toggweiler, J.R. 1992. On the structure and origin of 
major glaciation cycles. 1.- Linear responses to Milankovitch forcing. Paleoceanography 7, 701-738.

Jouet, G. 2007. Enregistrements stratigraphiques des cycles climatiques et glacio-eustatiques du Quaternaire terminal-Modélisations de la marge continentale du Golfe du Lion. Tesis doctoral. Université de Bretagne occidentale - Brest. Disponible en: http://tel.archives-ouvertes.fr/ tel-00260930/en/.

Lea, D.W., Martin, P.A., Pak, P.K., Spero, H.J. 2002. Reconstructing a 350 ky history of sea level using planktonic $\mathrm{Mg} / \mathrm{Ca}$ and oxygen isotope records from a Cocos Ridge core. Quaternary Science Reviews 21, 283-293.

Lisiecki, L.E., Raymo, M.E. 2005. A Pliocene-Pleistocene stack of 57 globally distributed benthic $\delta^{18} \mathrm{O}$ records. Paleoceanography 20, PA1003. Doi: 10.1029/2004PA001071.

Lobo, F.J., Tesson, M., Gensous, B. 2004. Stratral architectures of late Quaternary regressivetransgressive cycles in the Roussillon Shelf (SW Gulf of Lions, France). Marine and Petroleum Geology 21, 1181-1203.

Ludwig, W., Dumont, E., Meybeck, M., Heussner, S. 2009. River discharges of water and nutrients to the Mediterranean and Black Sea: Major drivers for ecosystem changes during past and future decades? Progress In Oceanography 80, 199-217.

Martinson, D.G., Pisias, N.G., Hays, J.D., Imbrie, J., Moore, T.C., Shackleton, N. 1987. Age dating and the orbital theory of the ice ages: development of a high-resolution 0 to 300,000-years chronostratigraphy. Quaternary Research 27, 1-29.

Miller, K.G., Kominz, M.A., Browning, J.V., Wright, J.D., Mountain, G.S., Katz, M.E., Sugarman, P. J., Cramer, B. S., Christie-Blick, N., Pekar, S. F. 2005. The Phanerozoic Record of Global Sea-Level Change. Science 310, 1293-1298.

NGRIP. 2004. High-resolution record of Northern Hemisphere climate extending into the last interglacial period. Nature 431, 147-151.

Palanques, A., Durrieu de Madron, X., Puig, P., Fabres, J., Guillén, J., Calafat, A., Canals, M., Heussner, S., Bonnin, J. 2006. Suspended sediment fluxes and transport processes in the Gulf of Lions submarine canyons. The role of storms and dense water cascading. Marine Geology 234, 43-61.

Pasqual, C., Sánchez-Vidal, A., Zúñiga, D., Calafat, A., Canals, M., Durrieu de Madron, X., Puig, P., Heussner, S., Palanques, A., Delsaut, N. 2010. Flux and composition of settling particles across the continental margin of the Gulf of Lion: the role of dense shelf water cascading. Biogeosciences 7, 217-231.

Pont, D., Simonnet, J.P., Walter, A.V. 2002. Medium-term Changes in Suspended Sediment Delivery to the Ocean: Consequences of Catchment Heterogeneity and River Management (Rhône River, France). Estuarine, Coastal and Shelf Science 54, 1-18.

Posamentier, H.W., Vail, P.R. 1988. Eustatic controls on clastic deposition II - sequence and systems tract models. En Sea-level changes: an integrated approach, C.G. Wilgus, B.S. Hastings, C.G.S.C. Kendall, H. Posamentier, C.A. Ross, J.C. Van Wagoner (eds.), Society of Economic Paleontologists and Mineralogists, Tulsa, Oklahoma, pp. 125-154.

Posamentier, H.W., Allen, G.P., James, D.P., Tesson, M. 1992. Forced regressions in a sequence stratigraphic framework: concepts, examples, and exploration significance. American Association of Petroleum Geologists (AAPG) Bulletin 76, 1687-1709.

Rabineau, M. 2001. Un modèle géométrique et stratigraphique des séquences de dépôts quaternaires de la plateforme du Golfe du Lion: enregistrement des cycles glacioeustatiques de 100000 ans. Tesis doctoral. Université de Rennes 1. Disponible en: http://archimer.ifremer.fr/search.jsp? record $=21$. 
Rabineau, M., Berné, S., Ledrezen, E., Lericolais, G., Marsset, T., Rotunno, M. 1998. 3D architecture of lowstand and transgressive Quaternary sand bodies on the outer shelf of the Gulf of Lion, France. Marine and Petroleum Geology 15, 439-452.

Rabineau, M., Berne, S., Aslanian, D., Olivet, J.L., Joseph, P., Guillocheau, F., Bourillet, J.F., Ledrezen, E., Granjeon, D. 2005. Sedimentary sequences in the Gulf of Lion: A record of 100,000 years climatic cycles. Marine and Petroleum Geology 22, 775-804.

Rohling, E.J., Grant, K., Bolshaw, M., Roberts, A.P., Siddall, M., Hemleben, C., Kucera, M. 2009. Antarctic temperature and global sea level closely coupled over the past five glacial cycles. Nature Geoscience 2, 500-504.

Ruddiman, W.F. 2003. Orbital insolation, ice volume, and greenhouse gases. Quaternary Science Reviews 22, 1597-1629.

Sánchez-Vidal, A., Canals, M., Calafat, A., Lastras, G., Pedrosa-Pàmies, R., Menéndez, M., Medina, R., Company, J.B., Hereu, B., Romero, J., Alcoverro, T. 2012. Impacts on the deep-sea ecosystem by a severe coastal storm. PLoS ONE 7 (1), e30395. Doi: 10.1371/journal.pone. 0030395.

Sánchez-Vidal, A., Pasqual, C., Kerhervé, P., Calafat, A., Heussner, S., Palanques, A., Durrieu de Madron, X., Canals, M., Puig, P. 2008. Impact of dense shelf water cascading on the transfer of organic matter to the deep western Mediterranean basin. Geophysical Research Letters 35. Doi:10.1029/2007GL032825, 5.

Shackleton, N.J., Hall, M.A., Vincent, E. 2000. Phase relationships between millennial-scale events 64,000-24,000 years ago. Paleoceanography 15, 565-569.

Siddall, M., Rohling, E.J., Alamogi-Labin, A., Hemleben, C., Meischner, D., Schmelzer, I., Smeed, D.A. 2003. Sea-level fluctuations during the last glacial cycle. Nature 423, 853-858.

Siddall, M., Chappell, J., Potter, E.K. 2006. Eustatic sea level during past interglacials. En The climate of past interglacials, F. Sirocko, T. Litt, M. Claussen, M. F. Sánchez Goñi (eds.), Elsevier, Amsterdam, pp. 75-92.

Siddall, M., Rohling, E.J., Thompson, W.G., Waelbroeck, C. 2008. MIS 3 sea level fluctuations: data synthesis and new outlook. Review of Geophysics 46, RG4003. Doi:10.1029/2007RG000226.

Sierro, F.J., Andersen, N., Bassetti, M.A., Berné, S., Canals, M., Curtis, J.H., Dennielou, B., Flores, J.A., Frigola, J., González-Mora, B., Grimalt, J.O., Hodell, D.A., Jouet, G., Pérez-Folgado, M., Schneider, R. 2009. Phase relationship between sea level and abrupt climate change. Quaternary Science Reviews 28, 2867-2881.

Svensson, A., Andersen, K.K., Bigler, M., Clausen, H.B., Dahl-Jensen, D., Davies, S.M., Johnsen, S.J., Muscheler, R., Parrenin, F., Rasmussen, S.O., Röthlisberger, R., Seierstad, I., Steffensen, J.P., Vinther, B.M. 2008. A 60,000 year Greenland stratigraphic ice core chronology. Climate of the Past 4, 47-57.

Tesson, M., Gensous, B., Allen, G.P., Ravenne, C. 1990. Late Quaternary deltaic lowstand wedges on the Rhône continental shelf, France. Marine Geology 91, 325-332.

Tesson, M., Posamentier, H.W., Gensous, B. 2000. Stratigraphic organization of late pleistocene deposits of the western part of the Golfe du Lion shelf (Languedoc shelf), Western Mediterranean Sea, using high-resolution seismic and core data. American Association of Petroleum Geologists (AAPG) Bulletin 84, 119-150.

Thompson, W.G., Goldstein, S.L. 2005. Open-System Coral Ages Reveal Persistent Suborbital Sea-Level Cycles. Science 308, 401-404.

Ulses, C., Grenz, V., Marsaleix, P., Schaaff, E., Estournel, C., Meulé, S., Pinazo, C. 2005. Circulation in a semi-enclosed bay under influence of strong freshwater input. Journal of Marine Systems 56, 113-132.

Vail, P.R., Mitchum, R.M., Todd, R.G., Widmier, J.M., Thompson, S., Sangree, J.B., Bubb, J.N., Hatleid, W.G. 1977. Seismic stratigraphy and global changes of sea level. En Seismic 
stratigraphy - applications to hydrocarbon exploration, C. E. Payton (ed.), The American Association of Petroleum Geologists, Tulsa, Oklahoma, pp. 49-212.

Van Kreveld, S.A., Sarnthein, M., Erlenkeuser, H., Grootes, P., Jung, S.J.A., Nadeau, M.J., Pflaumann, U., Voelker, A. 2000. Potential links between surging ice sheets, circulation changes, and the Dansgaard-Oeschger cycles in the Irminger Sea, 60-18 kyr. Paleoceanography 15, 425-442.

Voelker, A. 2002. Global distribution of centennial-scale records for marine isotope stage (MIS) 3: a database. Quaternary Science Reviews 21, 1185-1212.

Waelbroeck, C., Labeyrie, L., Michel, E., Duplessy, J.C., McManus, J.F., Lambeck, K., Balbon, E., Labracherie, M. 2002. Sea-level and deep water temperature changes derived from benthic foraminifera isotopic records. Quaternary Science Reviews 21, 295-305.

Yokoyama, Y., Esat, T.M., Lambeck, K. 2001. Coupled climate and sea-level changes deduced from Huon Peninsula coral terraces of the last ice age. Earth and Planetary Science Letters 193, 579-587. 